\title{
Systemic vasculitis in a patient with small-cell neuroendocrine bronchial cancer
}

\author{
T. Ponge*, D. Boutoille*, A. Moreau**, P. Germaud+, G. Dabouis*, T. Barangers, J. Barrier*
}

Systemic vasculitis in a patient with small-cell neuroendocrine bronchial cancer. T. Ponge, D. Boutoille, A. Moreau, P. Germaud, G. Dabouis, T. Baranger, J. Barrier. CERS Journals Ltd 1998.

ABSTRACT: A 75-yr-old male hospitalized for vascular purpura with joint pain had a medical history of polymyalgia rheumatica. A generalized oedematous syndrome occurred and the patient also presented with haemoptysis and complained of transient paraesthesia of the hands and feet. Renal biopsy showed lesions of focal segmental proliferative glomerulonephritis associated with a few cellular crescents. Lung biopsy showed small-cell neuroendocrine carcinoma. After the first course of chemotherapy signs of vasculitis disappeared.

Small-cell neuroendocrine carcinomas, which represent $25 \%$ of all lung cancers, have numerous paraneoplastic (especially neurological) extrapulmonary manifestations. Disseminated vasculitis has never been described with this type of cancer, whereas nonsmall-cell carcinomas are associated essentially with cutaneous vasculitis or purpura rheumatica. In the case reported here, anticancer chemotherapy allowed vasculitic manifestations to be treated.

Eur Respir J 1998; 12: 1228-1229.
*Médecine Interne $\mathrm{B}, * *$ Anatomie Pathologique, \#Oncologie Médicale Thoracique et Digestive, and \$Néphrologie, Hôtel-Dieu, Nantes, France. +Pneumologie, Hôpital Laënnec, Nantes, France.

Correspondence: T. Ponge, Médecine Interne B, Hôtel-Dieu, 44093 Nantes Cedex 1, France, Fax: 33240083309

Keywords: Nonsmall-cell lung cancer paraneoplastic vasculitis polymyalgia rheumatica purpura rheumatica

small-cell lung cancer

Received: January 221998

Accepted after revision July 51998
Vasculitis can be associated with cancers, most often in the form of blood disease but sometimes as solid tumours. However, this situation is rare, as indicated in a review of American literature that found only 11 cases involving solid tumours and 41 relating to blood disease [1]. Nonsmall-cell lung cancers can cause paraneoplastic syndromes which may be multiple (hypertrophic pulmonary osteoarthropathy and adrenocorticotrophic hormone secretion) in the same patient [2].

A case is reported of small-cell neuroendocrine bronchial carcinoma associated with disseminated vasculitis, which is a very rare occurrence for a type of cancer usually related to other forms of paraneoplastic syndrome [3].

\section{Case report}

A 75-yr-old male hospitalized for vascular purpura of the lower limbs had a medical history of polymyalgia rheumatica (PMR) diagnosed 8 months previously and treated with $6 \mathrm{mg}$ prednisone. The purpura, which appeared after surgery for an ordinary right inguinal hernia, recurred in association with joint pain. When it had nearly disappeared, a generalized oedematous syndrome occurred, with involvement of the lower limbs and loin, and bilateral pleural effusion. The patient also presented with haemoptysis and complained of transient paraesthesia of the hands and feet.

An inflammatory syndrome was observed, with an erythrocyte sedimentation rate of $110 \mathrm{~mm} \cdot \mathrm{h}^{-1}$, C-reactive protein $150 \mathrm{mg} \cdot \mathrm{L}^{-1}$, inflammatory normocytic normochromic anaemia (haemoglobin $6.6 \mathrm{~g} \cdot \mathrm{dL}^{-1}$ ) and lengthening of acti- vated partial thromboplastin time related to circulating anticoagulant. Plasma creatinine was $195 \mu \mathrm{mol} \cdot \mathrm{L}^{-1}$, with glomerular type proteinuria of $8 \mathrm{~g} \cdot 24 \mathrm{~h}^{-1}$ (with nephrotic syndrome). Three screenings for cryoglobulin were negative, as were those for antineutrophil cytoplasmic antibodies and antiglomerular basement membrane antibodies. Antinuclear antibodies were weakly positive (anti-SS-A of 74 (normal 0-30)). The myelogram showed nondystrophic normal lines. The biopsy revealed features of leukocytoclastic vasculitis, with a vascular deposit of immunoglobulin (Ig) A-C3 in immunofluorescence. The chest radiograph showed bilateral pleural effusion and a slight intraperitoneal effusion was visualized in abdominal ultrasonography. The muscle biopsy revealed simple fibre atrophy without

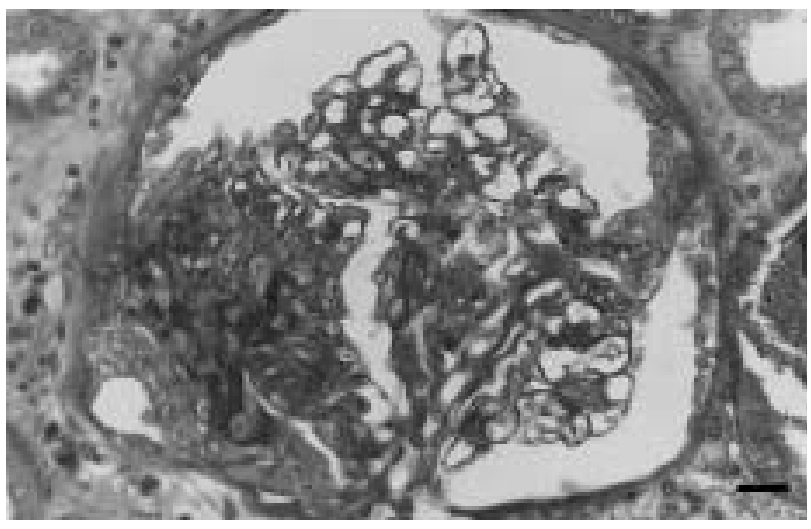

Fig. 1. - Renal biopsy trichrome staining showing focal segmental proliferative glomerulonephritis characterized by hypercellularity and mesangial enlargement, with the disappearance of capillary lumina. Note the segmental cellular crescent with fibrin. (Internal scale bar $=25 \mu \mathrm{m}$.) 


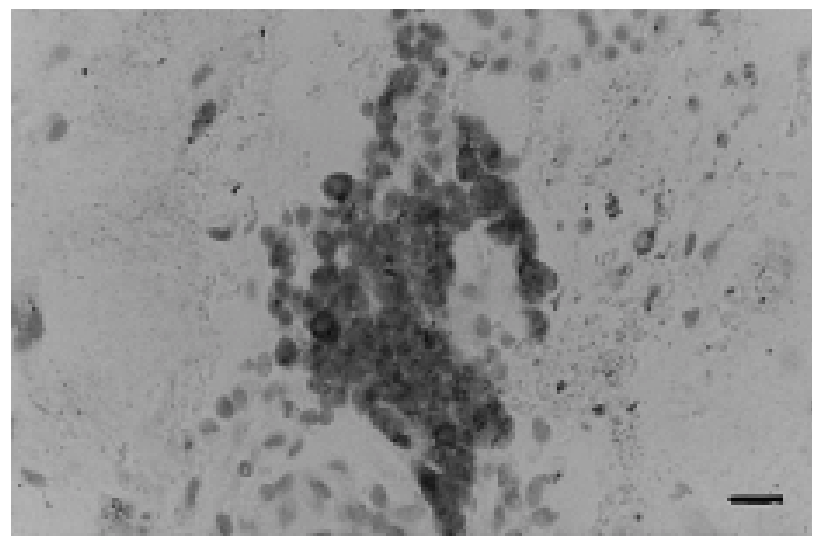

Fig. 2. - Bronchial biopsy. Immunohistochemical study with antisynaptophysin antibody showing strong cytoplasmic labelling of tumour cells. (Internal scale bar $=25 \mu \mathrm{m}$.)

specific vascular lesions. Renal arteriography was normal, whereas renal biopsy (fig. 1) showed lesions of focal segmental proliferative glomerulonephritis associated with a few cellular crescents. There was no significant glomerular fixation with immunofluorescence.

A bronchoscopic examination performed because of haemoptysis showed a macroscopic thickened and hyperaemic infiltration of the mucous membrane on the outer side of the lingula bronchus causing subtotal stenosis of this passage. Histological studies showed sheets of small cells with crushed nuclei within the bronchial chorion. The immunoreactivity of these atypical cells with antichromogranin A and antisynaptophysin antibodies (fig. 2) was indicative of small-cell neuroendocrine carcinoma. Neuron-specific enolase was $2.5 \mathrm{ng} \cdot \mathrm{mL}^{-1}$ (normal 0.0 12.5). Computed tomographic (CT) scans of the chest, abdomen and brain and bone scintigraphy suggested a cancer of the lingula localized in the chest.

Chemotherapy consisting of carboplatin and VP-16 was initiated, followed by chest radiotherapy (50 Gy in 25 sessions) focused on the mediastinum and the greater supraclavicular fossa. After the first course of chemotherapy, the signs of vasculitis disappeared and proteinuria dropped to $0.48 \mathrm{~g} \cdot 24 \mathrm{~h}^{-1}$.

\section{Discussion}

All of the patient's symptomatology (PMR, purpura, circulating anticoagulant and glomerulonephritis) was related to paraneoplastic vasculitis.

Associations between cancer and vasculitis have been reported, although sometimes in an anecdotal manner. The causal relation is difficult to prove, but is suggested by the parallel course of the two pathologies. Vasculitis generally precedes the discovery of cancer but can also occur afterwards. According to the registers of the University of Florida, there is a highly significant association between cutaneous vasculitis and myeloproliferative syndromes [4]. However, few studies have considered the relations between solid tumours and vasculitis. Four cases of purpura rheumatica (Schönlein-Henoch purpura) described during lung cancers had typical features: arthralgia involving several joints, abdominal pain, purpura and glomerulonephritis with IgA deposits. In the present case, the diagnosis of purpura rheumatica was not retained because of the absence of abdominal pain and glomerular IgA deposits.
Renal involvement of paraneoplastic origin (as suggested by a favourable course of the nephrotic syndrome after initial chemotherapy) usually takes the form of extramembranous glomerulonephritis when observed during stomach or lung cancers [5]. However, some cases of pure endocapillary glomerulonephritis have been reported during small-cell anaplastic carcinoma of the lung [6].

Paraneoplastic rheumatological manifestations are frequent. Four associations with PMR (polymyalgia rheumatica) and giant cell arteritis were found in a series of 45 patients [7]. Paraneoplastic PMR responds less well to corticosteroid therapy, which is quite rapidly effective in the case of primary PMR.

The existence of thromboembolic diseases during cancers has often been observed [1]. In the present case, circulating anticoagulant remained asymptomatic.

Small-cell neuroendocrine carcinomas represent around $25 \%$ of bronchopulmonary cancers. In addition to their metastatic potential, they are frequently characterized by paraneoplastic syndromes which are particularly apparent biologically, sometimes with the detection of autoantibodies [8]. To the authors' knowledge, systemic vasculitis has never been described before in association with this type of cancer.

The different manifestations of this patient are indicative of a context of systemic vasculitis developing gradually over an eight-month period and, as is often the case, preceding the diagnosis of cancer. However, different paraneoplastic syndromes could have been juxtaposed, as described in rare cases of nonsmall-cell lung cancer. Although spontaneous remission of vasculitis may have occurred independently of immunosuppressive treatment, this seems unlikely because of the prolonged course ( 8 months) and clinical severity.

The mechanism of these paraneoplastic syndromes is still uncertain but is probably multifactorial, involving the development of tumour-associated antigens responsible for circulating immunocomplexes and lymphokine production by the tumour.

\section{References}

1. Naschitz JE, Yeshurun D, Eldar S, et al. Diagnosis of cancer-associated disorders. Cancer 1996; 77: 1759-1767.

2. Monsieur I, Meysman M, Noppen M, et al. Nonsmallcell lung cancer with multiple paraneoplastic syndromes. Eur Respir J 1995; 8: 1231-1234.

3. van Osterhout AGM, van de Pol M, ten Velde GP, et al. Neurologic disorders in 203 consecutive patients with small cell lung cancer. Results of a longitudinal study. Cancer 1996; 77: 1434-1441.

4. Greer JM, Longley S, Edwards NL, et al. Vasculitis associated with malignancy. Experience with 13 patients and literature review. Medicine 1988; 67: 220-230.

5. Eagen JW, Lewis EJ. Glomerulopathies of neoplasia. Kidney Int 1977; 11: 297-306.

6. Jermanovich NB, Giammarco R, Ginsberg SJ, et al. Small cell anaplastic carcinoma of the lung with mesangial proliferative glomerulonephritis. Arch Intern Med 1982; 142: 397-399.

7. von Knorring J, Somer T. Malignancy in association with polymyalgia rheumatica and temporal arteritis. Scand $J$ Rheumatol 1974; 3: 129-135.

8. Dropcho EJ. Antiamphiphysin antibodies with small cell lung carcinoma and paraneoplastic encephalomyelitis. Ann Neurol 1996; 39: 659-667. 